\title{
Magnitud y Factores asociados a complicaciones de diabetes tipo 2: Análisis de un Sistema de Vigilancia de Diabetes Mellitus
}

\section{Magnitude and Factors associated with complications of type 2 diabetes: Analysis of a Diabetes Mellitus Surveillance System}

$\mathrm{DOI}$

https://doi.org/10.35434/rcmhnaaa.2021.143.1253

\section{RESUMEN}

Introducción: Las complicaciones por diabetes tipo 2 (DT2) están incrementando la demanda de servicios especializados en la Seguridad Social Peruana por lo que se torna necesario determinar la magnitud y factores asociados a complicación crónica por DT2 en esta población. Material y Métodos: Estudio transversal-analítico basado en 7875 registros de pacientes con DT2 $\geq 18$ años atendidos por consulta externa durante 2016-2017 y notificados en el Sistema de Vigilancia de Diabetes Mellitus de EsSalud. Se evaluaron variables sociodemográficas, clínicas y de comorbilidad mediante un análisis bivariado y un modelo de regresión de Poisson con varianza robusta para determinar la razón de prevalencia a complicación crónica por DT2. Resultados: La complicación microvascular de DT2 estuvo asociado a sexo masculino ( $R P=1,25 ; 95 \% I C: 1,10-1,42 ; p<0,01), H b A 1 c>9,0 \%$ (RP=1,25; 95\%IC: $1,08-1,46 ; p<0,01)$, hipertensión arterial $(R P=1,30 ; 95 \% I C: 1,14-1,49$; $p<0,01)$, anemia $(R P=1,57 ; 95 \% \mid C: 1,31-1,88 ; p<0,01)$, enfermedad tiroidea ( $R P=1,48 ; 95 \% I C: 1,07-2,03 ; p=0,02)$, cáncer ( $R P=1,70 ; 95 \% I C$ : $1,26-2,30 ; p<0,01)$ e hígado graso ( $R P=1,78 ; 95 \% I C: 1,28-2,48 ; p<0,01)$. El recibir educación para el autocuidado de DT2 mostró asociación negativa con complicación microvascular ( $\mathrm{RP}=0,84$; $95 \%$ IC: $0,73-0,96$; $p<0,01)$. La complicación macrovascular de DT2 estuvo asociado a sexo masculino ( $R P=1,52 ; 95 \% I C: 1,21-1,91 ; p<0,01)$, hipertensión arterial $(R P=2,72 ; 95 \% I C: 1,90-3,91 ; p<0,01)$, anemia $(R P=1,98 ; 95 \% I C: 1,33$ $2,95 ; \mathrm{p}<0,01)$ e hígado graso ( $R P=1,92 ; 95 \% I C: 1,03-3,62 ; p=0,04)$, mientras que la educación en DT2 mostró asociación negativa ( $R P=0,47$; 95\%IC: $0,36-0,61 ; p<0,01)$. Conclusiones: La educación en DT2 mostró asociación consistentemente con ausencia de complicación micro y macrovascular, sugiriendo ser un importante factor preventivo. Consideramos necesario evaluar el impacto de la educación mediante diseños apropiados.

Palabras Clave: Diabetes Mellitus Tipo 2; complicaciones de la diabetes; educación en salud; seguridad social (Fuente: DeCS-BIREME).

\section{ABSTRACT}

Background: Type 2 diabetes (DT2) complications has been increased the demand for specialized services in Peruvian Social Security(EsSalud), so it is necessary to determine the magnitude and factors associated with chronic complication due to DT2 in this population. Material and Methods: Cross-sectional study based on 7875 patients with DT2 $\geq 18$ years attended by ambulatory care during 2016-2017, notified in the Diabetes Mellitus Surveillance System of EsSalud. Sociodemographic, clinical and comorbidity variables were evaluated using a bivariate analysis and Poisson regression model with robust variance to determine the prevalence ratio of chronic
Víctor Cornetero-Muro, ${ }^{1, a}$, Nélida Hilario-Huapaya ${ }^{2,3, b}$, Jorge InolopúCucche $^{2,3 c}$, Cesar Ugarte-Gil ${ }^{2,4, a}$, Yamilée Hurtado-Roca ${ }^{5, a}$

FILIACIÓN

1. Gerencia Central de Prestaciones de Salud, Oficina de Inteligencia e Información Sanitaria, EsSalud. Lima, Perú.

2. Instituto de Medicina Tropical "Alexander von Humboldt", Universidad Peruana Cayetano Heredia. Lima, Perú.

3. Facultad de Salud Pública, Universidad Peruana Cayetano Heredia, Lima, Perú.

4. Facultad de Medicina, Universidad Peruana Cayetano Heredia, Lima, Perú

5. Instituto de Evaluación de Tecnologías en Salud e Investigación (IETSI), EsSalud, Lima, Perú

a. Médico Cirujano.

b. Licenciada en Enfermería.

c. Tecnólogo Médico.

ORCID

1. Víctor Cornetero-Muro / 0000-0002-9730-5067

2. Nélida Hilario-Huapaya / 0000-0001-9664-1803

3. Jorge Inolopú-Cucche / 0000-0002-2312-8387

5. Yamilée Hurtado-Roca / 0000-0003-1993-6223

CORRESPONDENCIA

Dr. Yamilée Hurtado-Roca

Instituto de Evaluación de Tecnologías en Salud e Investigación (IETSI), EsSalud. Lima, Perú.

Telf: 51 (1) 265-6000

EMAIL

lyhurtadoroca@gmail.com

CONFLICTOS DE INTERÉS

Los autores niegan conflictos de interés.

FINANCIAMIENTO

Esta investigación fue financiada por el Instituto de Evaluación de Tecnologías en Salud e Investigación (IETSI), EsSalud. Lima, Perú, mediante la adjudicación simplificada $\mathrm{N}^{\circ}$ 77-2017.

\section{AGRADECIMIENTO}

- Nélida Hilario es una estudiante de doctorado de Investigación Epidemiológica en la Universidad Peruana Cayetano Heredia becaria del FONDECYT/CIENCIACTIVA EF033235-2015 y es apoyada por el training grant D43TW007393 otorgado por el Fogarty International Center del US National Institutes of Health.

Agradecemos a la Dra. Joshi Acosta, al Dr. Víctor Suarez y al Dr. Manuel Catacora por su apoyo durante el proyecto.

REVISIÓN DE PARES

Recibido: $15 / 04 / 2021$

Aceptado: $16 / 08 / 2021$

\section{COMO CITAR}

Cornetero-Muro, V., Hilario-Huapaya, N., Inolopú-Cucche, J., Ugarte-Gil, C., \& Hurtado-Roca, Y. Magnitud y Factores asociados a complicaciones de diabetes tipo 2: Análisis de un Sistema de Vigilancia de Diabetes Mellitus. Revista Del Cuerpo Médico Hospital Nacional Almanzor Aguinaga Asenjo, 2021, 14(3). https://doi.org/10.35434/rcmhnaaa.2021.143.1253

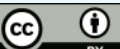

Esta obra está bajo una Licencia Creative Commons Atribución 4.0 Internacional. Versión Impresa: ISSN: 2225-5109 Versión Electrónica: ISSN: 2227-4731 Cross Ref. DOI: 10.35434/rcmhnaaa OJS: https://cmhnaaa.org.pe/ojs 
Revista del Cuerpo Médico del HNAAA, Vol 14 (3) - 2021

complication due to DT2. Results: The microvascular complication of DT2 was associated with male sex ( $P R=1.25 ; 95 \%$ Cl:1.10-1.42; $\mathrm{p}<0.01)$, HbA1c>9.0\% ( $P R=1.25 ; 95 \% \mathrm{Cl}: 1.08-1.46 ; \mathrm{p}<0.01)$, hypertension $(\mathrm{PR}=1.30 ; 95 \% \mathrm{Cl}: 1.14-1.49 ; \mathrm{p}<0.01)$, anemia $(\mathrm{PR}=1.57 ; 95 \% \mathrm{Cl}$ : 1.31-1.88; $p<0.01)$, thyroid disease $(P R=1.48 ; 95 \% C l: 1.07-2.03 ; p=0.02)$, cancer $(P R=1.70 ; 95 \% C l: 1.26-2.30 ; p<0.01)$ and fatty liver $(P R=1.78 ; 95 \% \mathrm{Cl}: 1.28-2.48 ; p<0.01)$. Receiving education for $\mathrm{DT} 2$ self-care showed a negative association with microvascular complication (RR=0.84; 95\% Cl:0.73-0.96; $\mathrm{p}<0.01$ ). DT2's macrovascular complication was associated with male sex ( $\mathrm{PR}=1.52 ; 95 \% \mathrm{Cl}: 1.21-1.91 ; \mathrm{p}$ $<0.01)$, arterial hypertension ( $P R=2.72 ; 95 \% \mathrm{Cl}: 1.90-3.91 ; \mathrm{p}<0.01)$, anemia $(\mathrm{PR}=1.98 ; 95 \% \mathrm{Cl}: 1.33-2.95 ; \mathrm{p}<0.01)$ and fatty liver $(\mathrm{PR}=1.92$; $95 \% \mathrm{Cl}: 1.03-3.62 ; \mathrm{p}=0.04$ ), while education in DT2 showed a negative association ( $\mathrm{PR}=0.47 ; 95 \% \mathrm{Cl}: 0.36-0.61 ; \mathrm{p}<0,01)$. Conclusions: Education in DT2 showed an association consistently with the absence of micro and macrovascular complications, suggesting that it is an important preventive factor. We consider it necessary to evaluate the impact of education through appropriate designs.

Keywords: Diabetes Mellitus, Type 2; Diabetes complications; Health Education; Social Security (Source: DeCS-BIREME).

\section{INTRODUCCIÓN}

Según la Federación Internacional de Diabetes; durante el 2017 la diabetes mellitus (DM) afectó a 425 millones de personas en el mundo y se espera que alcance 693 millones de casos para el año $2045^{(1)}$. El inadecuado control glicémico en DM está asociado al desarrollo de complicaciones crónicas de tipo micro y macrovascular, las cuales generan otras condiciones tales como ceguera, amputación, alteraciones gastrointestinales, insuficiencia arterial periférica, entre otras $^{(2)}$. La DM además está asociada a morbilidades tales como hipertensión arterial ${ }^{(3)}$, tuberculosis ${ }^{(4)}$ y depresión ${ }^{(5)}$. La morbilidad por DM conduce a una progresiva discapacidad que afectan la productividad laboral ${ }^{(6)}$ y generan gastos significativos en el sistema de salud ${ }^{(7,8)}$. Recientemente, el Seguro Social del Perú (EsSalud) ha implementado el Sistema de Vigilancia de Diabetes Mellitus de EsSalud (SVDM) con el objetivo de evaluar el acceso a los servicios de salud para la prevención y el manejo de las complicaciones en pacientes con DM. El presente estudio tiene como objetivo determinar la magnitud (frecuencia de DT2) y los factores asociados a complicación micro y macrovascular en población peruana con DT2 atendida en EsSalud mediante el análisis de registros del SVDM.

\section{MATERIALES Y MÉTODOS}

Realizamos un estudio transversal-analítico en pacientes con DT2 registrados en el SVDM con autorización institucional y aprobación del Comité de Ética de la Universidad Peruana Cayetano Heredia. Incluimos el total de registros de pacientes con edad $\geq 18$ años, diagnosticados con DT2 atendidos en los servicios de consulta externa de EsSalud durante los años 2016-2017. Las variables evaluadas fueron de tipo sociodemográfica, clínica, de comorbilidad, complicaciones crónicas de DT2 y resultados de parámetros de control. Esta última incluyó pruebas de laboratorio, medidas antropométricas y hemodinámicas; determinadas en un lapso no mayor a los 3 meses previos a la notificación. Las comorbilidades evaluadas fueron hipertensión arterial, obesidad, dislipidemia, anemia, enfermedad tiroidea, cáncer, hígado graso y tuberculosis activa (TB), con diagnóstico según el CIE-10. Las regiones de nacimiento y residencia fueron categorizadas en costa norte, costa centro, costa sur, sierra y selva, acorde a lo establecido por el Instituto Nacional de Estadística e Informática ${ }^{(9)}$. La variable cumplimiento del tratamiento fue notificada bajo criterio médico, la hospitalización previa tuvo como motivo la DT2 y la educación en DT2 consistió en haber recibido entrenamiento para el autocuidado. La complicación crónica microvascular fue clasificada en neuropatía, nefropatía, retinopatía y pie diabético; y la macrovascular en enfermedad arterial periférica, enfermedad cerebrovascular y enfermedad isquémica ${ }^{(2)}$. Considerando que las complicaciones crónicas microvasculares generalmente preceden a las macrovasculares, establecimos una clasificación basada en 2 grupos. El primero incluye a pacientes con complicación crónica microvascular pero sin complicación crónica macrovascular. El segundo incluye a pacientes complicación crónica macrovascular con o sin complicación crónica microvascular. En tal sentido, realizamos un análisis bivariado por pruebas paramétricas y no paramétricas para determinar los factores asociados a complicación micro y macrovascular. Además, desarrollamos un modelo de regresión de Poisson con varianza robusta para obtener razones de prevalencia (RP) $)^{(10)}$, que incluyó variables según criterio estadístico y epidemiológico. El análisis fue realizado con el software Stata 15 (Stata Corporation, College Station, TX, USA), y las gráficas mediante los software GraphPad Prism versión 7.00 (GraphPad Software, CA, USA) y Venny versión 2.1.0 (BioinfoGp-CNB, España) ${ }^{(11)}$.

\section{RESULTADOS}

Dispusimos de 7875 registros de pacientes con diagnóstico de DM durante los años 2016-2017 de los cuales descartamos 53 registros de pacientes menores de 18 años, 415 con diagnóstico diferente a DT2 y 45 duplicados; quedando un total de 7362 registros a ser analizados. La distribución de la población total con DT2, así como los grupos con y sin complicación crónica, fue descrita en relación a variables sociodemográficas y clínicas en la tabla 1. La población con DT2 se caracterizó por ser mayormente de sexo femenino $(53,6 \%)$, tener edad media de 64,8 años (desviación estándar (DE): 11,6$)$ e instrucción básica $(52,4 \%)$. La población de estudio proviene principalmente de la costa norte del Perú $(29,7 \%)$ y son predominantemente casos prevalentes $(90,8 \%)$. En relación con las condiciones clínicas, la población con DT2 presenta una mediana del tiempo de enfermedad y tratamiento de 8 años ( \pm rango intercuartil $(R I C): 13,0$ ) y 6 años ( \pm RIC: 11,0$)$, respectivamente. El tratamiento farmacológico fue principalmente mediante hipoglicemiantes orales $(68,3 \%)$ y solo el $18,1 \%$ alcanzó un óptimo control glicémico $(\mathrm{HbA} 1 \mathrm{c}<7 \%)$. 
Tabla 1. Características sociodemográficas y clínicas de pacientes con DT2 registrados en el sistema de vigilancia epidemiológica de EsSalud durante los años 2016 y 2017.

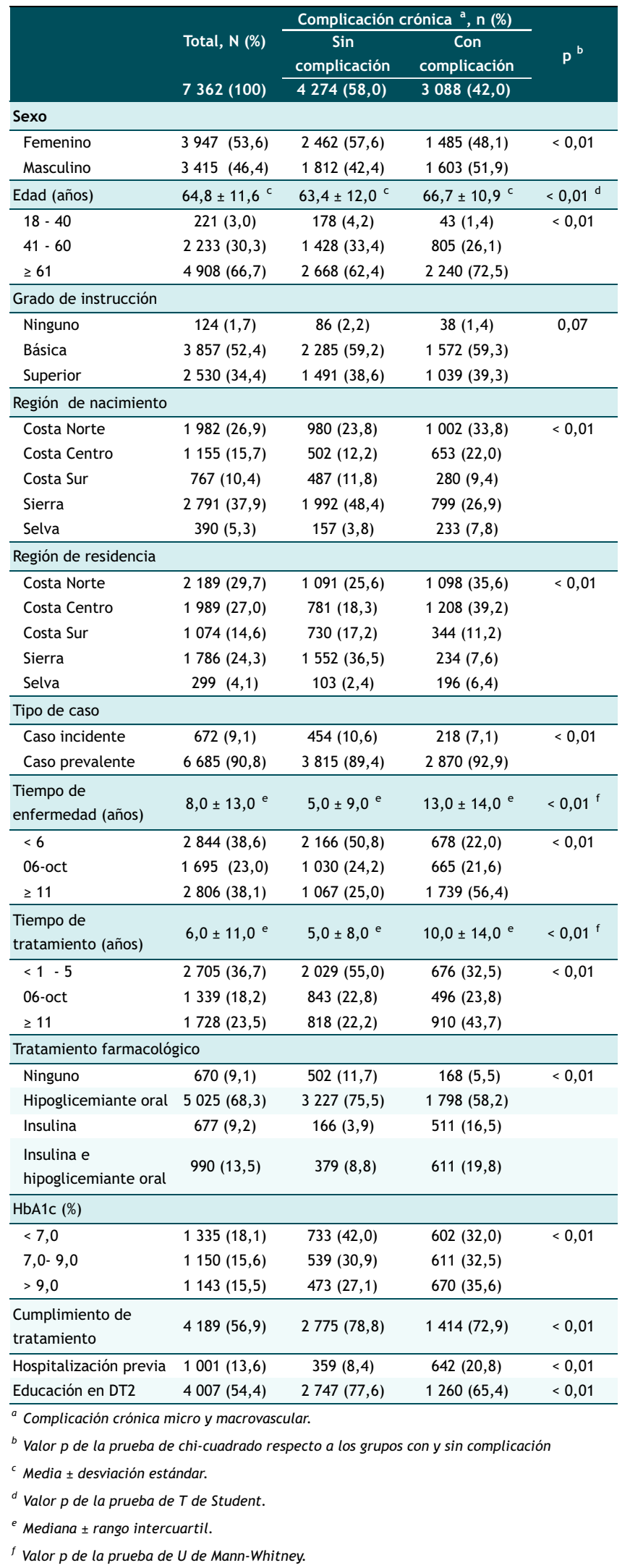

Tabla 2. Comorbilidad, complicaciones y parámetros de control de pacientes con DT2 registrados en el sistema de vigilancia epidemiológica de EsSalud durante los años 2016 y 2017.

\begin{tabular}{|c|c|c|c|c|}
\hline & \multirow[b]{2}{*}{ Total, N (\%) } & \multicolumn{2}{|c|}{ Complicación crónica $^{\mathrm{a}}, \mathrm{n}(\%)$} & \multirow{3}{*}{$p^{b}$} \\
\hline & & $\begin{array}{c}\text { Sin } \\
\text { complicación }\end{array}$ & $\begin{array}{c}\text { Con } \\
\text { complicación }\end{array}$ & \\
\hline & $7362(100)$ & $4274(58,0)$ & $3088(42,0)$ & \\
\hline Hipertensión arterial & $3728(50,6)$ & $1709(40,0)$ & $2019(65,4)$ & $<0,01$ \\
\hline Dislipidemia & $1362(18,5)$ & $740(17,3)$ & $622(20,1)$ & $<0,01$ \\
\hline Anemia & $355(4,8)$ & $73(1,7)$ & $282(9,1)$ & $<0,01$ \\
\hline Enfermedad tiroidea & $308(4,2)$ & $140(3,3)$ & $168(5,4)$ & $<0,01$ \\
\hline Cáncer & $211(2,9)$ & $86(2,0)$ & $125(4,1)$ & $<0,01$ \\
\hline Hígado graso & $181(2,5)$ & $73(1,7)$ & $108(3,5)$ & $<0,01$ \\
\hline Tuberculosis & $81(1,1)$ & $17(0,4)$ & $64(2,1)$ & $<0,01$ \\
\hline \multicolumn{5}{|c|}{ Categorías de índice de masa corporal $\left(\mathrm{kg} / \mathrm{m}^{2}\right)$} \\
\hline Infrapeso $(<19,0)$ & $51(0,7)$ & $22(0,6)$ & $29(1,3)$ & $<0,01$ \\
\hline Normopeso $(19,0-24,9)$ & $1201(16,3)$ & $659(18,3)$ & $542(25,1)$ & \\
\hline Sobrepeso $(25,0-29,9)$ & $2444(33,2)$ & $1559(43,3)$ & $885(41,0)$ & \\
\hline Obesidad $(\geq 30,0)$ & $2061(21,8)$ & $1357(37,7)$ & $704(32,6)$ & \\
\hline $\begin{array}{l}\text { Complicación crónica } \\
\text { microvascular }\end{array}$ & $2858(38,8)$ & NA & $2858(92,5)$ & NA \\
\hline Neuropatía diabética & $1845(25,1)$ & NA & $1845(59,7)$ & NA \\
\hline Nefropatía diabética & $1001(13,6)$ & NA & $1001(32,4)$ & NA \\
\hline Retinopatía diabética & $729(9,9)$ & NA & $729(23,6)$ & NA \\
\hline Pie diabético & $734(10,0)$ & NA & $734(23,8)$ & NA \\
\hline $\begin{array}{l}\text { Complicación crónica } \\
\text { macrovascular }\end{array}$ & $684(9,3)$ & NA & $684(22,1)$ & NA \\
\hline Enfermedad isquémica & $423(5,8)$ & NA & $423(13,7)$ & NA \\
\hline Enfermedad arterial periférica & $222(3,0)$ & NA & $222(7,2)$ & NA \\
\hline Enfermedad cerebro-vascular & $188(2,6)$ & NA & $188(6,1)$ & NA \\
\hline
\end{tabular}

Glicemia en ayunas $(\mathrm{mg} / \mathrm{dl})$

HbA1c (\%)

Colesterol total $(\mathrm{mg} / \mathrm{dl})$

$158,8 \pm 76,1^{c} 153,6 \pm 70,7^{c} 165,6 \pm 82,1^{c}<0,01^{d}$ $8,3 \pm 2,4^{c} \quad 8,0 \pm 2,4^{c} \quad 8,5 \pm 2,4^{c} \quad<0,01^{d}$ $183,5 \pm 71,5^{c} 192,3 \pm 83,5^{c} \quad 176,8 \pm 60,1^{c}<0,01^{c}$

Colesterol LDL $(\mathrm{mg} / \mathrm{dl})$

Colesterol HDL $(\mathrm{mg} / \mathrm{dl})$

Triglicéridos (mg/dl)

Microalbuminuria ( $\mathrm{mg} / 24$ horas)

Proteinuria (g/24 horas)

$101,5 \pm 47,4^{c} \quad 110,8 \pm 49,3^{c} \quad 94,2 \pm 44,6^{c}<0,01^{d}$

$45,8 \pm 21,3^{c} \quad 47,4 \pm 21,3^{c} \quad 44,8 \pm 21,2^{c}<0,01^{d}$ $178,4 \pm 120,8^{c} c^{182,2 \pm 122,0} 175,6 \pm 119,7^{c} \quad 0,14^{d}$

Índice de masa corporal $\left(\mathrm{kg} / \mathrm{m}^{2}\right)$ Perímetro abdominal en hombres (cm)

Perímetro abdominal en mujeres $(\mathrm{cm})$

Presión arterial sistólica (mmHg) $\quad 120,4 \pm 16,6^{c} \quad 118,4 \pm 15,7^{c} \quad 123,1 \pm 17,5^{c}<0,01^{d}$

Presión arterial diastólica

$(\mathrm{mmHg})$

$49,4 \pm 111,3^{c} 35,1 \pm 75,8^{c} \quad 68,5 \pm 143,4^{c}<0,01^{d}$

$90,0 \pm 169,5^{c} 59,0 \pm 119,8^{c} 106,3 \pm 188,8^{c}<0,01^{d}$

$28,8 \pm 5,0^{c} \quad 29,1 \pm 4,9^{c} \quad 28,3 \pm 5,2^{c} \quad<0,01^{c}$

$99,8 \pm 10,5^{c} \quad 99,7 \pm 10,2^{c} \quad 99,8 \pm 11,1^{c} \quad 0,97^{d}$

$98,3 \pm 12,2^{c} \quad 98,5 \pm 12,0^{c} \quad 97,9 \pm 12,6^{c} \quad 0,29^{d}$

${ }^{a}$ Complicación crónica micro y macrovascular.

${ }^{\mathrm{b}}$ Valor $\mathrm{p}$ de la prueba de chi-cuadrado respecto a los grupos con y sin complicación crónica, a ${ }^{\mathrm{C}}$ Media \pm desviación estándar.

${ }^{\text {d }}$ Valor $\mathrm{p}$ de la prueba de T de Student.

NA: No aplica.

La complicación crónica por DT2 se presentó en el 42,0\% y respecto al grupo sin complicación, tuvo significativa mayor frecuencia de pacientes de sexo masculino ( $51,9 \%$ vs $42,4 \%$; $\mathrm{p}<0,01)$ y mayor edad media $(66,7 \pm \mathrm{DE}: 10,9$ vs $63,4 \pm \mathrm{DE}$ : $12,0 ; p<0,01)$. Consecuentemente, la complicación crónica mostró mayor prevalencia en la costa norte del Perú (33,8\% vs $23,8 \%, p<0,01$ ), mayor mediana del tiempo de enfermedad $(13,0 \pm$ RIC: 14,0 vs 5,0 \pm RIC: 9,$0 ; p<0,01)$ y mayor tiempo de 
tratamiento $(10,0 \pm$ RIC: 14,0 vs $5,0 \pm$ RIC: 8,$0 ; p<0,01)$ Además, la población con complicación crónica presentó una significativa mayor frecuencia $\mathrm{HbA} 1 \mathrm{c}>9,0 \%(35,6 \%$ vs $27,1 \%$; $\mathrm{p}<0,01)$ y hospitalización previa $(20,8 \%$ vs $8,4 \% ; p<0,01)$. No obstante, esta tuvo menor frecuencia en el cumplimiento de tratamiento $(72,9 \%$ vs $78,8 \% ; p<0,01)$ y haber recibido educación en DT2 (65,4\% vs 77,6\%; $\mathrm{p}<0,01)$. De modo similar, la tabla 2 muestra la distribución de la población con DT2, así como los grupos con y sin complicación crónica, en relación a comorbilidad, tipo de complicación crónica y parámetros de control. La comorbilidad más frecuente en la población con DT2 fue hipertensión arterial $(50,6 \%)$, seguida de obesidad $(21,8 \%)$ y dislipidemia $(18,5 \%)$. Por su parte, la complicación microvascular representó el $38,8 \%$ de la población con DT2, siendo mayor la neuropatía $(25,1 \%)$, seguido de nefropatía $(13,6 \%)$, pie diabético $(10,0 \%)$ y retinopatía $(9,9 \%)$. Así mismo, la complicación macrovascular en la población con DT2 se presentó en el $9,3 \%$, siendo mayor la enfermedad isquémica $(5,8 \%)$, seguida de enfermedad arterial periférica $(3,0 \%)$ y enfermedad cerebrovascular $(2,6 \%)$.

Todas las comorbilidades evaluadas estuvieron asociadas a la complicación crónica $(p<0,01)$, a excepción de las categorías de sobrepeso y obesidad, que se presentaron con mayor frecuencia en el grupo sin complicación $(p<0,01)$. La complicación microvascular se presentó en el $92,5 \%$ de los casos de complicación crónica por DT2, y la complicación macrovascular en el $22,1 \%$. Se notificaron 454 registros de pacientes con complicación micro y macrovascular que representan el $66,4 \%$ de la complicación macrovascular y el $15,9 \%$ de los registros con complicación microvascular (figura 1). La tabla 2 también muestra las medias de los resultados de los parámetros de control en la población con DT2, que además fueron expresados en razón a sus valores referenciales (figura 2). Según el análisis bivariado, el grupo con complicación crónica, respecto al grupo sin complicación, tuvo significativo mayor valor de glicemia $(165,6 \pm$ DE: 82,1 vs $153,6 \pm D E: 70,7 ; p<0,01), \operatorname{HbA} 1 \mathrm{c}(8,5 \pm$ DE: 2,4 vs $8,0 \pm D E: 2,4 ; p<0,01)$, microalbuminuria $(68,5 \pm$

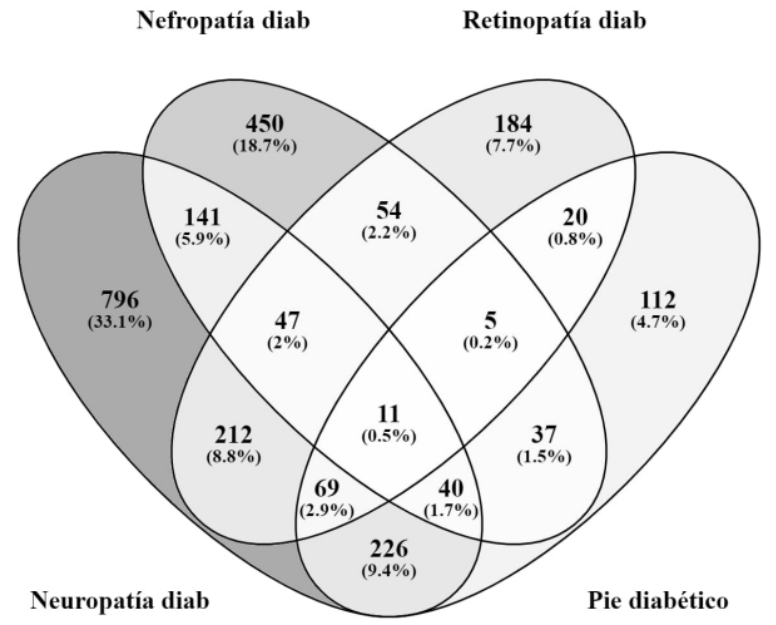

Figura 1.

Distribución de los tipos de complicaciones microvasculares en la población con DT2 registrada en el sistema de vigilancia (2016-2017).

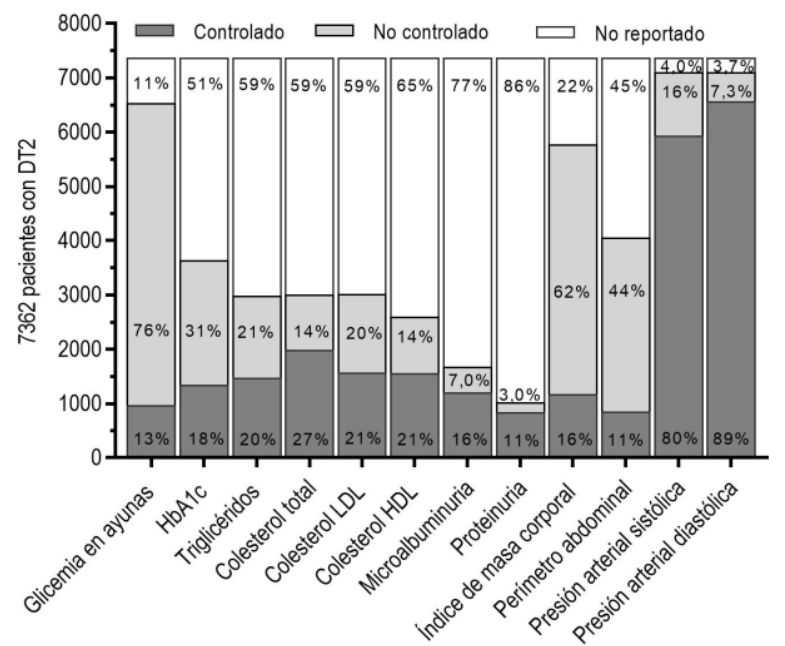

Figura 2.

Parámetros de control de DT2 en la población registrada en el sistema de vigilancia (2016-2017).

DE: 143,4 vs $35,1 \pm D E: 75,8 ; p<0,01$ ), entre otros. En contraste, el grupo con complicación crónica mostró menor nivel de colesterol total $(176,8 \pm \mathrm{DE}: 60,1$ vs $192,3 \pm \mathrm{DE}: 83,5$; $p<0,01)$, colesterol HDL $(44,8 \pm D E: 21,2$ vs $47,4 \pm D E: 21,3$; $p<0,01)$, colesterol LDL $(94,2 \pm D E: 44,6$ vs $110,8 \pm D E: 49,3$; $p<0,01)$ e IMC $(28,3 \pm D E: 5,2$ vs $29,1 \pm D E: 4,9 ; p<0,01)$.

La tabla 3 describe la distribución de los grupos (i) sin complicación, (ii) con complicación microvascular y (iii) complicación macrovascular, en relación a variables de interés previamente evaluadas. En tal sentido, los grupos con complicación micro y macrovascular respecto al grupo sin complicación, presentaron significativa mayor frecuencia de pacientes de sexo masculino, mayor edad media y procedencia de la costa norte del Perú $(p<0,01)$. Así mismo, ambos grupos de complicación crónica presentaron mayor tiempo de enfermedad, tiempo de tratamiento, uso de insulina y hospitalización previa $(p<0,01)$, acorde a los resultados anteriores. En contraste, las categorías de sobrepeso, obesidad, educación en DT2 y cumplimiento del tratamiento tuvieron significativa mayor frecuencia en el grupo sin complicación $(p<0,01)$. Con respecto a las comorbilidades evaluadas, todas mostraron asociación significativa con la complicación macrovascular respecto al grupo sin complicación $(\mathrm{p}<0,01)$. Sin embargo, únicamente la hipertensión arterial $(60,6 \%$ vs $40,0 \% ; p<0,01)$ y la anemia $(6,4 \%$ vs $1,7 \% ; p<0,01)$ tuvieron asociación significativa con complicación microvascular.

La tabla 4 muestra los resultados de un modelo de regresión de Poisson para determinar la RP de presentar complicación crónica microvascular y macrovascular. En el modelo crudo de la complicación microvascular, casi todas las variables evaluadas mostraron asociación significativa con presencia de complicación $(p<0,01)$, excepto las categorías de sobrepeso, obesidad y educación en DT2, que tuvieron asociación con ausencia de complicación $(p<0,01)$. Las variables dislipidemia, enfermedad tiroidea, cáncer e hígado graso no mostraron asociación en el modelo crudo $(p>0,05)$. En el modelo ajustado, el sexo masculino ( $R P=1,25$; 95\% IC: 
Tabla 3. Análisis bivariado de factores asociados a complicación crónica microvascular y macrovascular de pacientes con DT2 registrados en el sistema de vigilancia epidemiológica de EsSalud durante los años 2016 y 2017.

\begin{tabular}{|c|c|c|c|c|c|}
\hline & \multirow{2}{*}{$\begin{array}{c}\text { Sin } \\
\text { complicación, } \\
\mathrm{n}(\%)\end{array}$} & \multicolumn{3}{|c|}{ Complicación crónica, n (\%) } & \multirow{3}{*}{$p^{c}$} \\
\hline & & $\begin{array}{l}\text { Complicación } \\
\text { microvascular } \\
\text { a }\end{array}$ & & $\begin{array}{l}\text { Complicación } \\
\text { macrovascular } \\
\text { b }\end{array}$ & \\
\hline & $4274(58,0)$ & $2404(32,6)$ & $p^{c}$ & $684(9,4)$ & \\
\hline \multicolumn{6}{|l|}{ Sexo } \\
\hline Femenino & $2462(57,6)$ & $1199(49,9)$ & $<0,01$ & $286(41,8)$ & $<0,01$ \\
\hline Masculino & $1812(42,4)$ & $1205(50,1)$ & & $398(58,2)$ & \\
\hline Edad (años) & $63,4 \pm 12,0^{d}$ & $65,7 \pm 10,8^{d}$ & $<0,01{ }^{\mathrm{e}}$ & $70,3 \pm 10,4^{d}$ & $<0,01^{\mathrm{e}}$ \\
\hline $18-40$ & $178(4,2)$ & $43(1,8)$ & $<0,01$ & $0(0,0)$ & $<0,01$ \\
\hline $41-60$ & $1428(33,4)$ & $680(28,3)$ & & $125(18,3)$ & \\
\hline$\geq 61$ & $2668(62,4)$ & $1681(69,9)$ & & $559(81,7)$ & \\
\hline \multicolumn{6}{|l|}{ Región de residencia } \\
\hline Costa Norte & $1091(25,6)$ & $760(31,7)$ & $<0,01$ & $338(49,6)$ & $<0,01$ \\
\hline Costa Centro & $781(18,4)$ & $944(39,4)$ & & $264(38,7)$ & \\
\hline Costa Sur & $730(17,1)$ & $288(12,0)$ & & $56(8,2)$ & \\
\hline Sierra & $1552(36,5)$ & $223(9,3)$ & & $11(1,6)$ & \\
\hline Selva & $103(2,4)$ & $183(7,6)$ & & $13(1,9)$ & \\
\hline $\begin{array}{l}\text { Tiempo de enfermedad } \\
\text { (años) }\end{array}$ & $5,0 \pm 9,0^{f}$ & $13,0 \pm 14,0^{f}$ & $<0,01^{\mathrm{g}}$ & $14,0 \pm 14,5^{f}$ & $<0,01^{\mathrm{s}}$ \\
\hline$<1-5$ & $2166(50,8)$ & $528(22,0)$ & $<0,01$ & $150(21,9)$ & $<0,01$ \\
\hline 06-oct & $1030(24,2)$ & $522(21,8)$ & & $143(20,9)$ & \\
\hline$\geq 11$ & $1167(25,0)$ & $1348(56,2)$ & & $391(57,2)$ & \\
\hline $\begin{array}{l}\text { Tiempo de tratamiento } \\
\text { (años) }\end{array}$ & $5,0 \pm 8,0^{f}$ & $10,0 \pm 13,0^{f}$ & $<0,01^{\mathrm{g}}$ & $10,0 \pm 14,0^{f}$ & $<0,01^{\mathrm{g}}$ \\
\hline$<1-5$ & $2029(55,0)$ & $539(32,7)$ & $<0,01$ & $137(31,7)$ & $<0,01$ \\
\hline 06-oct & $843(22,8)$ & $393(23,8)$ & & $103(23,8)$ & \\
\hline$\geq 11$ & $818(22,2)$ & $718(43,5)$ & & $192(44,5)$ & \\
\hline \multicolumn{6}{|l|}{ Tratamiento } \\
\hline Ninguno & $502(11,7)$ & $120(5,0)$ & $<0,01$ & $48(7,0)$ & $<0,01$ \\
\hline Hipoglicemiante oral & $3227(75,5)$ & $1426(59,3)$ & & $372(54,5)$ & \\
\hline $\begin{array}{l}\text { Insulina e } \\
\text { hipoglicemiante oral }\end{array}$ & $379(8,9)$ & $452(18,8)$ & & $159(23,2)$ & \\
\hline Insulina & $116(3,9)$ & $406(16,9)$ & & $105(15,3)$ & \\
\hline \multicolumn{6}{|l|}{ HbA1c (\%) } \\
\hline$<7,0$ & $733(42,0)$ & $441(30,8)$ & $<0,01$ & $161(35,9)$ & 0,05 \\
\hline $7,0-9,0$ & $539(30,9)$ & $452(31,5)$ & & $159(35,4)$ & \\
\hline$>9,0$ & $473(27,1)$ & $541(37,7)$ & & $129(28,7)$ & \\
\hline \multicolumn{6}{|c|}{ Categorías de índice de masa corporal $\left(\mathrm{kg} / \mathrm{m}^{2}\right)$} \\
\hline Infrapeso $(<19,0)$ & $22(0,61)$ & $22(1,29)$ & $<0,01$ & $7(1,52)$ & $<0,01$ \\
\hline $\begin{array}{l}\text { Normopeso }(19,0 \text { - } \\
24,9)\end{array}$ & $659(18,3)$ & $429(25,2)$ & & $113(24,6)$ & \\
\hline $\begin{array}{l}\text { Sobrepeso }(25,0- \\
29,9)\end{array}$ & $1559(43,3$ & $705(41,5)$ & & $180(39,1)$ & \\
\hline Obesidad $(\geq 30,0)$ & $1357(37,7)$ & $544(32,0)$ & & $160(34,8)$ & \\
\hline Educación en DT2 & $2747(77,6)$ & $1057(69,5)$ & $<0,01$ & $203(49,7)$ & $<0,01$ \\
\hline $\begin{array}{l}\text { Cumplimiento de } \\
\text { tratamiento }\end{array}$ & $2775(78,8)$ & $1124(73,3)$ & $<0,01$ & $290(71,4)$ & $<0,01$ \\
\hline Hospitalización previa & $359(8,4)$ & $439(18,3)$ & $<0,01$ & $203(29,7)$ & $<0,01$ \\
\hline Hipertensión arterial & $1709(40,0)$ & $1457(60,6)$ & $<0,01$ & $562(82,2)$ & $<0,01$ \\
\hline Dislipidemia & $740(17,3)$ & $420(17,5)$ & 0,87 & $202(29,5)$ & $<0,01$ \\
\hline Anemia & $73(1,7)$ & $153(6,4)$ & $<0,01$ & $129(18,9)$ & $<0,01$ \\
\hline Enfermedad tiroidea & $140(3,3)$ & $91(3,8)$ & 0,27 & $77(11,3)$ & $<0,01$ \\
\hline Cáncer & $86(2,0)$ & $57(2,4)$ & 0,33 & $68(9,9)$ & $<0,01$ \\
\hline Hígado graso & $73(1,7)$ & $56(2,3)$ & 0,08 & $52(7,6)$ & $<0,01$ \\
\hline Tuberculosis & $17(0,4)$ & $14(0,6)$ & 0,29 & $50(7,3)$ & $<0,01$ \\
\hline
\end{tabular}

${ }^{a}$ Neuropatia, retinopatia, nefropatia, y/o pie diabético.
${ }^{b}$ Enfermedad arterial periférica, enfermedad cerebro-vascular y/o enfermedad isquémica; con o $\sin$ presencia de complicación microvascular.

'Prueba chi-cuadrado respecto a los grupos con complicación crónica y el grupo sin complicación, a menos que se indique lo contrario.

${ }^{d}$ Media \pm desviación estándar.

Valor p de la prueba de $T$ de Student.

Mediana \pm rango intercuartil.

${ }^{s}$ Valor $p$ de la prueba de $U$ de Mann-Whitney.
1,10-1,42; $p<0,01)$, tiempo de enfermedad $(R P=1,02 ; 95 \% I C$ : $1,01-1,03 ; p<0,01)$ y $\mathrm{HbA} 1 \mathrm{C}>9,0 \%(\mathrm{RP}=1,25 ; 95 \% \mathrm{IC}: 1,08-$ $1,46 ; \mathrm{p}<0,01)$ mostraron asociación con complicación microvascular. En contraste, el sobrepeso ( $R P=0,82$; $95 \% \mathrm{IC}$ : $0,70-0,96 ; p=0,02)$, obesidad ( $R P=0,81 ; 95 \% I C: 0,68-0,96$; $\mathrm{p}=0,02)$ y educación en DT2 ( $R P=0,84 ; 95 \% I C$ : $0,73-0,96$; $\mathrm{p}<0,01)$ mostraron asociación con ausencia de complicación microvascular. Las comorbilidades de hipertensión arterial $(R P=1,30 ; 95 \% I C: 1,14-1,49 ; p<0,01)$, anemia $(R P=1,57$; 95\%IC: $1,31-1,88 ; p<0,01)$, enfermedad tiroidea $(R P=1,48$; $95 \% I C: 1,07-2,03 ; p=0,02)$, cáncer $(R P=1,70 ; 95 \% I C: 1,26$ $2,30 ; p<0,01)$ e hígado graso ( $R P=1,78 ; 95 \% I C: 1,28-2,48$; $\mathrm{p}<0,01)$ también mostraron asociación con complicación microvascular. Por otro lado, la complicación macrovascular en el modelo crudo mostró asociación positiva con todas las variables evaluadas, excepto con educación en DT2, sobrepeso, obesidad y educación en DT2, las cuales estuvieron asociadas significativamente con no presentar complicación crónica $(\mathrm{p}<0,01)$. En el modelo ajustado, el sexo masculino ( $R P=1,52 ; 95 \% \mathrm{IC}: 1,21-1,91 ; p<0,01)$, edad $(R P=1,03 ; 95 \% I C: 1,02-1,04 ; p<0,01)$, tiempo de enfermedad $(\mathrm{RP}=1,02 ; 95 \% \mathrm{IC}: 1,00-1,03 ; \mathrm{p}<0,01)$, hipertensión arterial $(R P=2,72 ; 95 \% I C: 1,90-3,91 ; p<0,01)$, anemia $(R P=1,98$; 95\%IC: $1,33-2,95 ; p<0,01)$ e hígado graso $(R P=1,92 ; 95 \% I C:$ $1,03-3,62 ; p=0,04)$ tuvieron asociados a complicación macrovascular. De modo similar a lo presentado en el modelo de complicación microvascular; la educación en DT2 $(\mathrm{RP}=0,47 ; 95 \% \mathrm{IC}: 0,36-0,61 ; \mathrm{p}<0,01)$ estuvo asociada a no presentar complicación.

\section{DISCUSIÓN}

El estudio evaluó la magnitud y factores asociados a complicación crónica micro y macrovascular por DT2 en pacientes atendidos en EsSalud, constituyendo este el primer reporte de DT2 en población asegurada del Perú. Los principales resultados muestran asociación significativa con sexo masculino, edad avanzada, mayor tiempo de enfermedad, inadecuado control glicémico y ausencia de educación para el control de DT2. Respecto al control glicémico, niveles de $\mathrm{HbA} 1 \mathrm{c}>9,0 \%$ mostraron asociación significativa con complicación microvascular, probablemente debido a la baja adherencia al tratamiento con hipoglicemiantes o falta de recursos ${ }^{(12)}$. En el Perú, los establecimientos de primer nivel de atención generalmente no cuentan con médicos endocrinólogos, equipo multidisciplinario entrenado y pruebas de laboratorio para el monitoreo de la enfermedad. Ello es relevante desde una perspectiva de prevención de complicaciones secundarias de DT2, considerando además que una efectiva reducción del riesgo de complicación renal y ocular debe procurar mantener niveles de $\mathrm{HbA} 1 \mathrm{c}<7,0 \%{ }^{(13)}$. Por otro lado, es conocido que la obesidad está asociados al desarrollo de complicaciones micro y macrovasculares por DT2 ${ }^{(14)}$, recomendándose incluso en algunos casos procedimientos quirúrgicos como la cirugía bariátrica ${ }^{(15)}$. Sin embargo, nuestros resultados muestran que tanto el sobrepeso y obesidad están asociadas con ausencia de complicación lo cual parece ser contradictorio. No obstante, en estudios de corte transversal la asociación entre IMC y el riesgo de mortalidad en pacientes con DM no es clara, considerando además la presencia de factores confusores, uso del IMC como indicador de obesidad o causalidad reversa ${ }^{(16)}$. 
Tabla 4. Análisis bivariado y multivariado de factores asociados a complicación crónica micro y macrovascular de pacientes con DT2 registrados en el sistema de vigilancia epidemiológica de EsSalud durante los años 2016 y 2017.

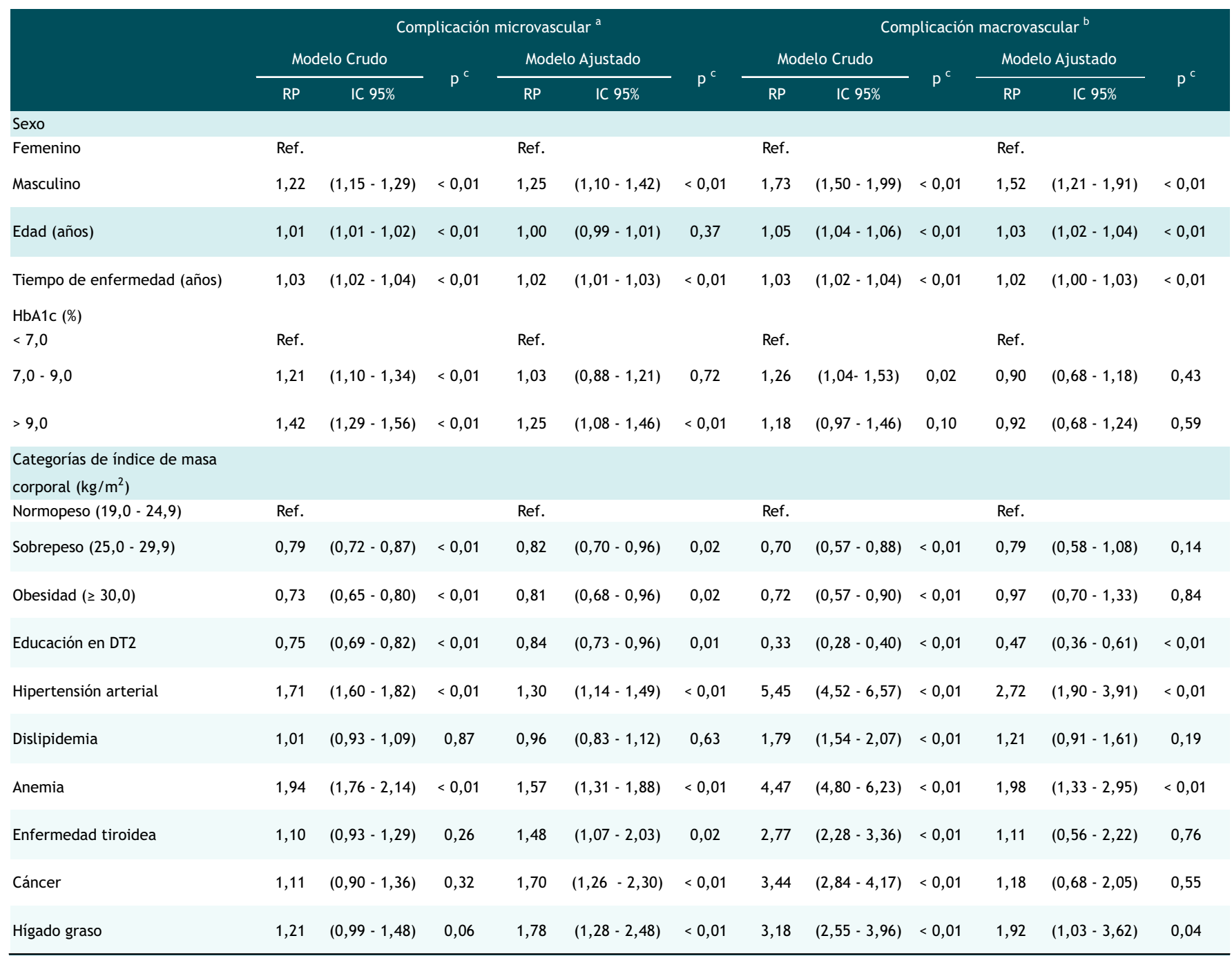

Neuropatía, retinopatía, nefropatía, y/o pie diabético; sin complicación microvascular.

${ }^{b}$ Enfermedad arterial periférica, enfermedad cerebro-vascular y/o enfermedad isquémica; con o sin presencia de complicación microvascular.

valor $p$ del modelo de regresión de Poisson con varianza robusta. El modelo ajustado incluye a todas las variables presentes en la tabla.

Nuestro estudio determinó que la educación en DT2 para el autocuidado estuvo asociado consistentemente con ausencia de complicación micro y macrovascular, coincidiendo con los esfuerzos de EsSalud en el desarrollo del Programa Integral del Asegurado con $\mathrm{DM}^{(17)}$. Un estudio realizado en pacientes peruanos pertenecientes en una región con alta prevalencia de DT2 identificó que la participación en talleres educativos incrementó significativamente el nivel de conocimientos y prácticas de autocuidado ${ }^{(18)}$. Consideramos necesario evaluar la posibilidad de implementar programas educativos que permita detectar oportunamente complicaciones por DT2 para evitar su progresión a ceguera, pie diabético, entre otros $^{(19)}$. Estos programas pudieran estar enfocados al perfil del paciente según su condición de cronicidad pues sus necesidades educativas son diferentes. Wong y col., desarrollaron un programa de educación estructurado para la DM que mostró eficacia en la reducción de los niveles de HbA1c, mortalidad por diversas causas y previno los primeros eventos de enfermedad micro y macrovascular en pacientes con DT2 ${ }^{(20)}$.

Respecto a las comorbilidades evaluadas, la hipertensión arterial, anemia, enfermedad tiroidea, cáncer e hígado graso mostraron asociación significativa con complicación microvascular. La hipertensión arterial es una de las comorbilidades más relevantes en DM debido a su elevada mortalidad y relación con la complicación micro y macrovascular en DT2. Nuestro estudio reportó un $60,6 \%$ de prevalencia de hipertensión arterial entre los casos con complicación microvascular. Cabe precisar que la ADA considera hipertensión arterial con niveles $\geq 140 / 90 \mathrm{mmHg}$ de presión arterial ${ }^{(21)}$ al igual que los estándares actuales en Perú( ${ }^{(22)}$. Sin embargo, otros contextos como la Asociación Austriaca de DM, consideran la hipertensión arterial a niveles $\geq 130 / 80 \mathrm{mmHg}^{(23)}$. Dado que la coexistencia de hipertensión arterial y DM incrementa el riesgo de enfermedad cardiovascular ateroesclerótica, la Asociación Americana del Corazón $(\mathrm{AHA})$ recomienda también niveles $\geq 130 / 80$ 
Revista del Cuerpo Médico del HNAAA, Vol 14 (3) - 2021

$\mathrm{mmHg}\left(24^{)(2)}\right.$. Estas discordancias son importantes abordarlas pues ello podría generar error de clasificación. Acorde a los resultados de Asher y col. ${ }^{(3)}$ nuestros resultados muestran que control glucémico deficiente, la mayor duración de DM y la presencia de hipertensión arterial estuvo asociado con complicación microvascular.

En cuanto a la anemia, esta es una complicación comúnmente asociada con duración de la $\mathrm{DM}^{(25)}$ y está vinculado a la enfermedad renal crónica. Por ello, si bien hay que considerar que es un marcador de daño renal, los niveles de hemoglobina reducidos se pueden asociar también en pacientes con mayor riesgo de complicación microvascular, enfermedad cardiovascular y mortalidad ${ }^{(26)}$. Los niveles de hemoglobina en pacientes con DM podrían brindar información útil en prevenir complicaciones micro y macrovasculares por $\mathrm{DM}^{(27)}$. El estudio también identificó asociación entre enfermedad tiroidea y complicaciones microvasculares. Según las Guías Clínicas de Endocrinología (AME) y de Diabetología (AMD) italianas, la enfermedad tiroidea y DM no muestran asociación consistente ${ }^{(28)}$. No obstante, estos indican que los pacientes con DT2 y alteración en la función tiroidea presentan alto riesgo de complicación cardiovascular y microvascular propias de la DM. En tal un estudio muestra que los pacientes con DT2 y valores incrementados de TSH presentan mayor prevalencia de nefropatía diabética ${ }^{(29)}$. Así mismo, estudios en población coreana con DT2, existe una ligera asociación entre hipotiroidismo subclínico y retinopatía diabética severa ${ }^{(30)}$.

Nuestros resultados también mostraron asociación entre cáncer y complicación microvascular. La ADA destaca que la DM incrementa el riesgo de cáncer de hígado, páncreas, endometrio, colon/recto, mama y vejiga, pero con escasa la evidencia. A pesar de ello, la ADA recomienda educar a los pacientes en factores de riesgo y de incentivar la detección oportuna ${ }^{(21)}$. Al respecto, un estudio identificó que la razón neutrófilo- linfocito (NLR) era 1,14 veces mayor en pacientes con DM con al menos una complicación microvascular, siendo el NLR un conocido indicador en algunos tipos de cáncer $^{(31)}$. Acorde a ello, un estudio identificó a las enfermedades cardiovasculares y el cáncer entre las principales causas de muerte en $\mathrm{DM}^{(32)}$.

Por su parte, hígado graso resultó asociado a complicación microvascular. Resultado que es coherente con los resultados de Afarideh y col., quienes identificaron que el diagnóstico de hígado graso no alcohólico (HGNA) con o sin elevación de enzimas hepáticas séricas se correlaciona inversamente con complicación crónica microvascular. Entre sus explicaciones a este hallazgo se plantea causalidad inversa, por adaptación fisiológica compensatoria ${ }^{(33)}$. Otro estudio en pacientes coreanos identificó que el HGNA se asocia inversamente con prevalencias de retinopatía y nefropatía diabética ${ }^{(34)}$.

En relación a la complicación macrovascular, esta se asoció con sexo masculino, edad, tiempo de enfermedad, hipertensión arterial, anemia e hígado graso. Un estudio identificó que la edad fue un factor de riesgo para complicaciones macro vasculares y que la aparición de enfermedad arterial coronaria, neuropatía periférica, pie diabético y amputación de extremidad inferior fue significativamente mayor entre los hombres que en mujeres ${ }^{(35)}$. Sobre hipertensión arterial, los datos son congruentes con los estudios encontrados en la literatura, debido a que por diferentes vías patogénicas que incluyen hiperglucemia y resistencia a la insulina se puede generar complicaciones macro vasculares, por lo que es comprensible su asociación ${ }^{(36,37)}$. Respecto a anemia, un estudio realizado en China, identificó tasas más altas de anemia ante complicaciones micro y macro vasculares, y en su análisis encontró que los pacientes con anemia tienen 2,10 veces el riesgo de presentar complicación macrovascular ${ }^{(27)}$. Existe información respecto a DM e HGNA, entre los que destacan que ante la presencia de ambas enfermedades existe incremento del riesgo de desarrollar complicaciones micro y macrovascular. Así mismo que existe mayor riesgo de mortalidad por todas las causas y causas específicas de tipo cardiovascular, cáncer e hígado, en comparación con aquellos sin $\mathrm{HGNA}^{(38)}$.

Nuestro estudio presenta algunas limitaciones debido la naturaleza del análisis de base de datos secundaria, existiendo posibilidad de sesgo de información debido a que hubieron datos faltantes, sobre todo en factores como $\mathrm{HbA1C}$, colesterol y proteinuria que llegaron al rango entre $50 \%-80 \%$, Cabe destacar que esta limitación fue difícil de controlar debido a que los datos de las variables ya habían sido recolectados por el personal de salud como parte de la práctica clínica y no con fines de investigación. Por ello, existe la posibilidad de sesgo no diferencial, que podría llevar a sub-estimación de la asociación encontrada. Cabe remarcar que la base analizada corresponde a datos nacionales y es la más grande de población asegurada con DT2, por lo que a pesar de las limitaciones permite un primer acercamiento a la magnitud real de las complicaciones por DT2. Así mismo, podría haber confusores inadecuadamente medidos o no reconocidos, sin embargo, se ha realizado revisión detallada de la literatura para identificarlos y reducir este riesgo.

En conclusión, la complicación crónica micro y macrovascular en individuos con DT2 está asociada fundamentalmente con educación en DT2. Las complicaciones microvasculares estuvieron asociadas al sexo masculino, mayor tiempo de enfermedad, $\mathrm{HbA} 1 \mathrm{c}>9 \%$, tener sobrepeso y obesidad, hipertensión arterial, anemia, enfermedad tiroidea, cáncer e hígado graso. Las complicaciones macrovasculares se asocia con sexo masculino, edad, tiempo de enfermedad, hipertensión arterial, anemia e hígado graso. Debido a que el estudio fue análisis de base de datos secundaria y se identificó dificultad para enlazar las diferentes fuentes de información, se hacen necesarios estudios prospectivos que permitan ampliar estos resultados.

\section{REFERENCIAS BIBLIOGRÁFICAS}

1. Cho NH, Shaw JE, Karuranga S, Huang Y, da Rocha Fernandes JD, Ohlrogge AW, et al. IDF Diabetes Atlas: Global estimates of diabetes prevalence for 2017 and projections for 2045. Diabetes Res Clin Pract. 2018;138:271-81. doi: 10.1016/j.diabres.2018.02.023.

2. Harding JL, Pavkov ME, Magliano DJ, Shaw JE, Gregg EW. Global trends in diabetes complications: a review of current evidence. Diabetologia. 2019;62(1):3-16. doi: 10.1007/s00125-018-4711-2.

3. Lastra G, Syed S, Kurukulasuriya LR, Manrique C, Sowers JR. Type 2 diabetes mellitus and hypertension: an update. Endocrinol Metab Clin North Am. 2014;43(1):103-22. doi: 10.1016/j.ecl.2013.09.005.

4. Sarker M, Barua M, Guerra F, Saha A, Aftab A, Latif AH, et al. Double Trouble: Prevalence and Factors Associated with Tuberculosis and Diabetes Comorbidity in Bangladesh. Plos one. 
2016;11(10):e0165396. doi: 10.1371/journal.pone.0165396.

5. Luppens D, Piette C, Radermecker RP, Scantamburlo G, Ansseau M, Pitchot W. [Depression and type 2 diabetes: etiopathogenic analysis of a frequent comorbidity]. Rev Med Liege [Internet]. 2014 [cited 2021 April 5];69(11):611-7. Available from: https://orbi.uliege.be/handle/2268/174578.

6. Ramsey S, Summers KH, Leong SA, Birnbaum HG, Kemner JE, Greenberg P. Productivity and medical costs of diabetes in a large employer population. Diabetes Care. 2002;25(1):23-9. doi: 10.2337/diacare.25.1.23.

7. Seuring T, Archangelidi O, Suhrcke M. The Economic Costs of Type 2 Diabetes: A Global Systematic Review. Pharmacoeconomics. 2015;33(8):811-31. doi: 10.1007/s40273-015-0268-9.

8. Arredondo A. Type 2 diabetes and health care costs in Latin America: exploring the need for greater preventive medicine. BMC Med. 2014;12:136. doi: 10.1186/s12916-014-0136-z.

9. Obregon G, Zevallos K, Alarcon V, Puyen ZM, Chavez Inagaki O, Mendoza-Ticona A, et al. Rapid drug susceptibility testing and treatment outcomes for multidrug-resistant tuberculosis in Peru. Int J Tuberc Lung Dis. 2018;22(11):1350-7. doi: 10.5588/ijtld.17.0894.

10. Coutinho LM, Scazufca M, Menezes PR. Methods for estimating prevalence ratios in cross-sectional studies. Rev Saude Publica [Internet]. 2008 [cited 2021 April 10];42(6):992-8. Available from: https://www.scielo.br/j/rsp/a/XdMZzxdGrSTS77Y8ZgpGdWj/?lang $=$ en.

11. Oliveros JC. Venny [Internet]. An interactive tool for comparing lists with Venn's diagrams 2007-2015. [citado el 11 de abril de 2021]. D i s p o n i bl e http://bioinfogp.cnb.csic.es/tools/venny/index.html.

12. Barra Malig SF Adherencia al tratamiento farmacológico oral de personas con diabetes mellitus en 7 localidades de la Costa de Perú. [Tesis]. Lima: Repositorio Institucional Universidad Peruana Cayetano Heredia; 2018 [citado el 10 de abril de 2021]. Disponible en: https: / / hdl.handle.net/20.500.12866/3737.

13. Zoungas S, Arima H, Gerstein HC, Holman RR, Woodward M, Reaven P, et al. Effects of intensive glucose control on microvascular outcomes in patients with type 2 diabetes: a meta-analysis of individual participant data from randomised controlled trials. Lancet Diabete Endocrinol. 2017;5(6):431-7. doi: 10.1016/S2213-8587(17)30104-3.

14. Shera AS, Jawad F, Maqsood A, Jamal S, Azfar M, Ahmed U. Prevalence of chronic complications and associated factors in type 2 diabetes. J Pak Med Assoc. 2004;54(2):54-9.

15. Sheng B, Truong K, Spitler H, Zhang L, Tong X, Chen L. The Long-Term Effects of Bariatric Surgery on Type 2 Diabetes Remission, Microvascular and Macrovascular Complications, and Mortality: Systematic Review and Meta-Analysis. Obes Surg. 2017;27(10):2724 32. doi: 10.1007/s11695-017-2866-4

16. Han SJ, Boyko EJ. The Evidence for an Obesity Paradox in Type 2 Diabetes Mellitus. Diabetes Metab J. 2018;42(3):179-87. doi: 10.4093/dmj.2018.0055.

17. Gerencia de Prestaciones de Salud - EsSalud. Cuidado integral del asegurado con diabetes mellitus en el Seguro Social de Salud - EsSalud [Internet]. Lima, Perú; 2016 [citado el 12 de abril de 2021]. D i s p o n i b l e

e $n$ https://ww1.essalud.gob.pe/compendio/pdf/0000003397_pdf.pdf.

18. Red Asistencial La Libertad, EsSalud. Efecto de talleres de prevención y autocuidado sobre los niveles de conocimientos y prácticas en personas con diabetes Mellitus tipo 2 [Internet]. La Libertad: Red Asistencial La Libertad, EsSalud; 2014. [citado el 12 de abril de 2021]. D i s p o n i ble e n : h t t p : / / w w w http://repositorio.essalud.gob.pe/jspui/bitstream/ESSALUD/7/1/e fecto_prev_autocuidado_diabetes.pdf.

19. Li J, Gu L, Guo Y. An educational intervention on foot self-care behaviour among diabetic retinopathy patients with visual disability and their primary caregivers. J Clin Nurs. 2019;28(13-14):2506-2516. doi: $10.1111 /$ jocn. 14810 .

20. Wong CK, Wong WC, Wan EY, Chan AK, Chan FW, Lam CL. Macrovascular and microvascular disease in obese patients with type 2 diabetes attending structured diabetes education program: a population-based propensity-matched cohort analysis of Patient Empowerment Programme (PEP). Endocrine. 2016;53(2):412-22. doi: $10.1007 / \mathrm{s} 12020-015-0843-z$.

21. American Diabetes Association. Standards of medical care in diabetes
- 2019. J Clin Appl Res Educ. 2019;42(1):1-204

22. Dirección de Prevención de Enfermedades No transmisibles y Oncológicas. Guía de Práctica Clínica para el Diagnóstico, Tratamiento y Control de la Diabetes Mellitus Tipo 2 en el Primer Nivel de Atención [Internet]. Lima: R.M. N 719-2015/MINSA; 2016 [citado el 13 de abril de 2021 . Disponible en: http://bvs.minsa.gob.pe/local/MINSA/3466.pdf.

23. Schernthaner G, Saely $\mathrm{CH}$, Schernthaner G-H, Watschinger B, Drexel HJWkW. Individualisierung der antihypertensiven Therapie bei Patienten mit Diabetes mellitus. Leitlinie der Österreichischen Diabetes Gesellschaft (Update 2019). Wien Klin Wochenschr. 2019; 131(Suppl 1):124-135. doi: 10.1007/s00508-019-1460-2.

24. Shin D, Bohra C, Kongpakpaisarn K. Impact of the Discordance Between the American College of Cardiology/American Heart Association and American Diabetes Association Recommendations on Hypertension in Patients With Diabetes Mellitus in the United States. $\mathrm{H}$ y p e r t e n sion. $2018 ; 72$ ( 2): $256-9$. d o i : 10.1161/HYPERTENSIONAHA.118.11422.

25. Hosseini MS, Rostami Z, Saadat A, Saadatmand SM, Naeimi E. Anemia and microvascular complications in patients with type 2 diabetes mellitus. Nephrourol Mon. 2014;6(4):e19976. doi: 10.5812/numonthly. 19976.

26. Thomas MC. Anemia in diabetes: marker or mediator of microvascular disease? Nat Clin Pract Nephrol. 2007;3(1):20-30. doi: 10.1038/ncpneph0378.

27. He BB, Xu M, Wei L, Gu YJ, Han JF, Liu YX, et al. Relationship between Anemia and Chronic Complications in Chinese Patients with Type 2 Diabetes Mellitus. Arch Iran Med. 2015;18(5):277-83.

28. Guastamacchia E, Triggiani V, Aglialoro A, Aiello A, Ianni L, Maccario $M$, et al. Italian Association of Clinical Endocrinologists (AME) \& Italian Association of Clinical Diabetologists (AMD) Position Statement : Diabetes mellitus and thyroid disorders: recommendations for clinical practice. Endocrine. 2015;49(2):33952. doi: 10.1007/s12020-014-0474-9.

29. Qi Q, Zhang QM, Li CJ, Dong RN, Li JJ, Shi JY, et al. Association of Thyroid-Stimulating Hormone Levels with Microvascular Complications in Type 2 Diabetes Patients. Med Sci Monit. 2017;23:2715-20. doi: 10.12659/MSM.902006.

30. Gao ZH, Yuan RY, Li GP. Comment on: "Association between subclinical hypothyroidism and severe diabetic retinopathy in Korean patients with type 2 diabetes". Endocr J. 2011;58(12):1105-6. doi: 10.1507/endocrj.ej11-0281.

31. Dar MS, Beg SA. TNM cancer staging: can it help develop a novel staging system for type 2 diabetes? Diabetes Metab Syndr Obes. 2018;11:845-53. doi: 10.2147/DMSO.S179963.

32. Asfandiyarova N, Kolcheva N, Ryazantsev I, Ryazantsev V. Risk factors for stroke in type 2 diabetes mellitus. Diab Vasc Dis Res. 2006;3(1):5760. doi: $10.3132 /$ dvdr.2006.009.

33. Afarideh M, Aryan Z, Ghajar A, Ganji M, Ghaemi F, Saadat M, et al. Association of non-alcoholic fatty liver disease with microvascular complications of type 2 diabetes. Prim Care Diabetes. 2019;13(6):505-514. doi: 10.1016/j.pcd.2019.03.009.

34. Kim BY, Jung CH, Mok JO, Kang SK, Kim CH. Prevalences of diabetic retinopathy and nephropathy are lower in Korean type 2 diabetic patients with non-alcoholic fatty liver disease. J Diabetes Investig. 2014;5(2):170-5. doi: 10.1111/jdi.12139.

35. Arambewela MH, Somasundaram NP, Jayasekara HBPR, Kumbukage MP, Jayasena PMS, Chandrasekara CMPH, et al. Prevalence of Chronic Complications, Their Risk Factors, and the Cardiovascular Risk Factors among Patients with Type 2 Diabetes Attending the Diabetic Clinic at a Tertiary Care Hospital in Sri Lanka. J Diabetes Res. 2018; 2018:4504287. doi: 10.1155/2018/4504287.

36. Viigimaa M, Sachinidis A, Toumpourleka M, Koutsampasopoulos K, Alliksoo S, Titma T. Macrovascular complications of type 2 diabetes mellitus. Curr Vasc Pharmacol. 2020;18(2):110-116. doi: 10.2174/1570161117666190405165151.

37. de Havenon A, Majersik JJ, Tirschwell DL, McNally JS, Stoddard G, Rost NS. Blood pressure, glycemic control, and white matter hyperintensity progression in type 2 diabetics. Neurology. 2019;92(11):e1168-e75. doi: 10.1212/WNL.0000000000007093.

38. Tilg $H$, Moschen AR, Roden M. NAFLD and diabetes mellitus. Nat Rev Gastroenterol Hepatol. 2017;14(1):32-42. doi: $10.1038 /$ nrgastro.2016.147. 